\title{
Pharmacist-Coordinated Multidisciplinary Hospital Follow-up Visits Improve Patient Outcomes
}

\author{
Jamie J. Cavanaugh, PharmD, CPP, BCPS; Kimberly N. Lindsey, PharmD; \\ Betsy B. Shilliday, PharmD, CDE, CPP, BCACP; and Shana P. Ratner, MD
}

\begin{abstract}
BACKGROUND: The Affordable Care Act of 2010 allows for the adjustment of reimbursement to health care centers based on 30-day readmission rates. High readmission rates may be explained by multiple events at discharge, including medication errors that occur during the transition of care from inpatient to outpatient. Pharmacist involvement at discharge has been shown to improve health outcomes in patients with chronic disease; however, there is limited knowledge regarding the benefits of a clinic appointment with a pharmacist postdischarge.
\end{abstract}

OBJECTIVE: To compare hospital readmission rates and interventions in a multidisciplinary team visit coordinated by a clinical pharmacist practitioner with those conducted by a physician-only team within an internal medicine hospital follow-up program.

METHODS: A retrospective observational study was completed. Patients seen between May 2012 and January 2013 in 1 of the 2 hospital followup program models (multidisciplinary team or physician-only team) were included.

RESULTS: A total of 140 patient visits were included for 124 patients. Patients seen by the multidisciplinary team had a 30 -day readmission rate of $14.3 \%$ compared with $34.3 \%$ by the physician-only team ( $P=0.010)$. Interventions completed during the visits, including addressing nonadherence, initiating a new medication, and discontinuing a medication were also statistically different between the groups, with the multidisciplinary team completing these interventions more frequently.

CONCLUSIONS: Hospital follow-up visits coordinated by the multidisciplinary team decreased 30-day hospital readmission rates compared with follow-up visits by a physician-only team.

J Manag Care Spec Pharm. 2015;21(3):256-60

Copyright @ 2015, Academy of Managed Care Pharmacy. All rights reserved.

\section{What is already known about this subject}

Pharmacist involvement at discharge and postdischarge follow-up phone calls are beneficial in improving adherence and decreasing hospital readmission rates, respectively.

Pharmacist involvement during postdischarge clinic visits has been associated with a decrease in 60-day hospital readmission rates.

\section{What this study adds}

Standardized multidisciplinary hospital follow-up visits, coordinated by a clinical pharmacist practitioner, had a lower 30-day readmission rate than physician-only visits.

Interventions such as addressing adherence, initiating medications, and discontinuing medications may be contributing factors to lower readmission rates seen in the multidisciplinary team model.

1 harmacist involvement in managing patients with chronic disease has been shown to improve health outcomes. ${ }^{1-3}$ However, few published studies document the role of a face-to-face clinic visit with a pharmacist in the transition from hospital to outpatient care. A smooth transition of care is especially important in improving the well-being of patients and decreasing preventable hospital readmissions. One in five Medicare patients are readmitted within 30 days. ${ }^{4}$ The 2010 Affordable Care Act calls for reduction of payment to hospitals with 30-day readmission rates higher than the average riskadjusted readmission rates, potentially resulting in significant financial repercussions for health care centers.

Multiple errors may occur during the transition from inpatient to outpatient care, especially related to medication therapy. These errors include discontinuation of prescribed medications; noncompliance, including failure to fill medications or failure to be adherent; and delays in filling medications..$^{5-7}$ In the geriatric population, the most frequently reported unwanted events after discharge were related to prescription medication regimens mainly due to patients getting incorrect drugs or dosages. ${ }^{8}$

Literature to date shows that some pharmacist interventions before and after discharge decrease medication-related errors. Pharmacist counseling before discharge improves overall medication adherence after discharge from $34.8 \%$ to $55.2 \%$ when compared with standard of care (i.e., information session with a nurse). ${ }^{9}$ Pharmacist counseling at discharge and via phone within 3 to 5 days of discharge reduced preventable, medication-related emergency department visits or hospital readmissions from $8 \%$ to $1 \%$ when compared with usual care, which included medication review by a pharmacist and counseling from a nurse. ${ }^{10}$ Patients receiving general medication 
therapy management by a clinical pharmacist postdischarge had a 60 -day readmission rate of $18.2 \%$ compared with $43.1 \%$ who did not have a clinic visit with a pharmacist. ${ }^{11}$ While evidence suggests that inpatient pharmacists at discharge and ambulatory care pharmacists after discharge improve adherence and/or decrease preventable hospital readmissions, little is known about the role and impact of the outpatient ambulatory care pharmacist as part of a multidisciplinary team postdischarge. Little is also known about the impact of an ambulatory care pharmacist as part of a multidisciplinary team on decreasing 30-day hospital readmission rates.

A pilot hospital follow-up program began seeing patients in the University of North Carolina (UNC) Internal Medicine Clinic in March 2012. The goal was to improve the transition of care for patients being discharged from UNC Hospitals and to decrease the hospital readmission rate from a baseline of 19\% (based on February 2012 Medicaid data) by seeing patients in the clinic within 7 days of discharge. Patients could be referred by the care manager, or other provider, or scheduled based on an internally developed risk stratification status independent of reasons for admission or payer status. The care manager sought to first schedule moderate- and high-risk patients and then offered appointments to low-risk patients if appointments were available. During each hospital follow-up clinic visit, the purpose was to complete a thorough medication review, as well as address lifestyle interventions and barriers to care. Other visit components included a thorough patient history, a physical exam as appropriate, ordering of laboratory tests and medications if needed, and educating patients on self-management and symptoms needing immediate medical attention. ${ }^{12}$

There were 2 models in the hospital follow-up program: a multidisciplinary team model and a physician-only team model. The multidisciplinary team included a clinical pharmacist practitioner (CPP) and an attending physician. Within the state of North Carolina, CPPs are licensed pharmacists who are recognized as advanced practice providers. CPPs may prescribe medication therapy and order appropriate monitoring tests within an agreed upon protocol and under the supervision of an attending physician. The physician-only team included a medical resident and an attending physician. The initial intent of the physician-only team was to provide additional learning opportunities for medical residents and expand clinic access. The medical residents were trained by the pharmacist to perform the tasks required for the program and were provided a standard program template outlining the visit components. Both team types received assistance from a care manager in scheduling the appointments and addressing barriers to care, such as transportation and obtaining medications prior to the visit. The care manager was also available to both team types during the clinic visit as needed. The same group of attending physicians saw patients with the CPP and the residents.
The multidisciplinary team saw patients 7 half-days per week, and the physician-only team saw patients on 2 different half-days of the week to maximize overall clinic availability. Patients were scheduled to be seen by the multidisciplinary team or the physician-only team based on the patient's preferred appointment time. The difference in team structure was not explained to the patient prior to scheduling.

The overall hospital follow-up program, regardless of team type, has been shown to prevent one 30-day hospital readmission for every 7 patients seen, an absolute risk reduction of $16.7 \%$, when compared with usual care. ${ }^{12}$ While it is clear that the program as a whole has improved transitions, it is unknown if there is any difference in 30-day hospital readmission rates between the 2 distinct team types carrying out the intervention.

The purpose of this study was to compare the transitions of care, measured by hospital readmission rates and medication interventions, in a multidisciplinary team visit coordinated by a CPP with those conducted by a physician-only team in a hospital follow-up program.

\section{Methods}

After the establishment of the hospital follow-up program, a retrospective review of the 2 hospital follow-up models was conducted using an integrated electronic health record system that included inpatient and outpatient records. More patient visits were completed by the multidisciplinary team than the physician-only team during the study period. To maintain an equal number of patient visits in each study group, all patients meeting the inclusion criteria seen by the physician-only team were included. Patients seen by the multidisciplinary team were first matched to the physician-only team patients by discharge date and then selected at random. If a patient selected at random did not meet the inclusion criteria, the next random patient was reviewed for inclusion until an equal number of patient visits were selected for each team type within the matched time period.

\section{Inclusion/Exclusion Criteria}

A convenience sample of patients discharged from UNC Hospitals and seen in the UNC Internal Medicine Clinic followup program between May 2012 and January 2013 were included in this study. Patients were excluded if they were admitted for planned chemotherapy, same-day gastrointestinal procedures, discharged to hospice, or discharged to skilled nursing facilities.

\section{Study Variables}

The primary endpoint was 30-day hospital readmission at UNC Hospitals. The secondary endpoint was improvement in transition of care as measured by interventions such as frequency of medication discontinuation, recommendation of lower cost alternatives, initiation of new medications, identification of and 
addressing nonadherence, identification of wrong duration of medication, and medication dose adjustment. Fisher's exact test was used to determine if there was a difference between the 2 groups for the primary and secondary endpoints.

\section{Results}

Data were collected on a total of 140 patient visits $(n=70$ patient visits per group) completed by the hospital follow-up program. A total of 78 patient visits were completed by the physician-only team during the study time period, 8 of which did not meet inclusion criteria. A total of 311 patient visits were completed by the multidisciplinary team, 241 of which were not included after matching by discharge date to patients in the physician-only group, random selection to maintain equal group size, and meeting exclusion criteria. Four patients in the physician-only team group and 3 patients in the multidisciplinary team group had more than 1 visit (from more than 1 hospital discharge) and were counted as separate patient visits. There were 8 patients seen by both team types once during the study time period. Patients with a visit from both team types were included as separate patient visits in each team group.

The majority of patients were female, and the median age was 57 years (Table 1). Patients in each group had similar characteristics, including length of hospital stay and number of medications and medication changes at discharge. The number of days between hospital discharge and hospital follow-up visit were also similar between groups, with the majority of patients seen within 1 week. The physician-only team completed more patient visits with patients who were considered moderate and high risk for readmission compared with the multidisciplinary group based on the internal UNC readmission risk stratification.

\section{Primary/Secondary Endpoints}

For primary endpoints, patients seen by the multidisciplinary team had a 30-day readmission rate of $14.3 \%$ compared with $34.3 \%$ in the physician-only team $(P=0.010)$. For secondary endpoints, medication interventions were similar between the 2 groups for medication dose adjustments (37.9\% multidisciplinary team vs. $33.8 \%$ physician-only team, $P=0.719)$ and recommendation of a lower cost alternative $(0 \%$ multidisciplinary team vs. $2.9 \%$ physician-only team, $P=0.497$ ). The multidisciplinary team more frequently addressed nonadherence $(98.5 \%$ multidisciplinary team vs. $86.8 \%$ physician-only team, $P=0.017)$, initiated a new medication $(60.9 \%$ multidisciplinary team vs. $37.7 \%$ physician-only team, $P=0.010)$, and discontinued a medication (31.4\% multidisciplinary team vs. $15.7 \%$ physician-only team, $P=0.046)$.

\section{Subgroup Analysis}

A subgroup analysis including only patients at moderate or high risk for hospital readmission was completed to mitigate the potential difference in hospital readmission risk between the 2 groups. A total of 42 patient visits completed by the mul-

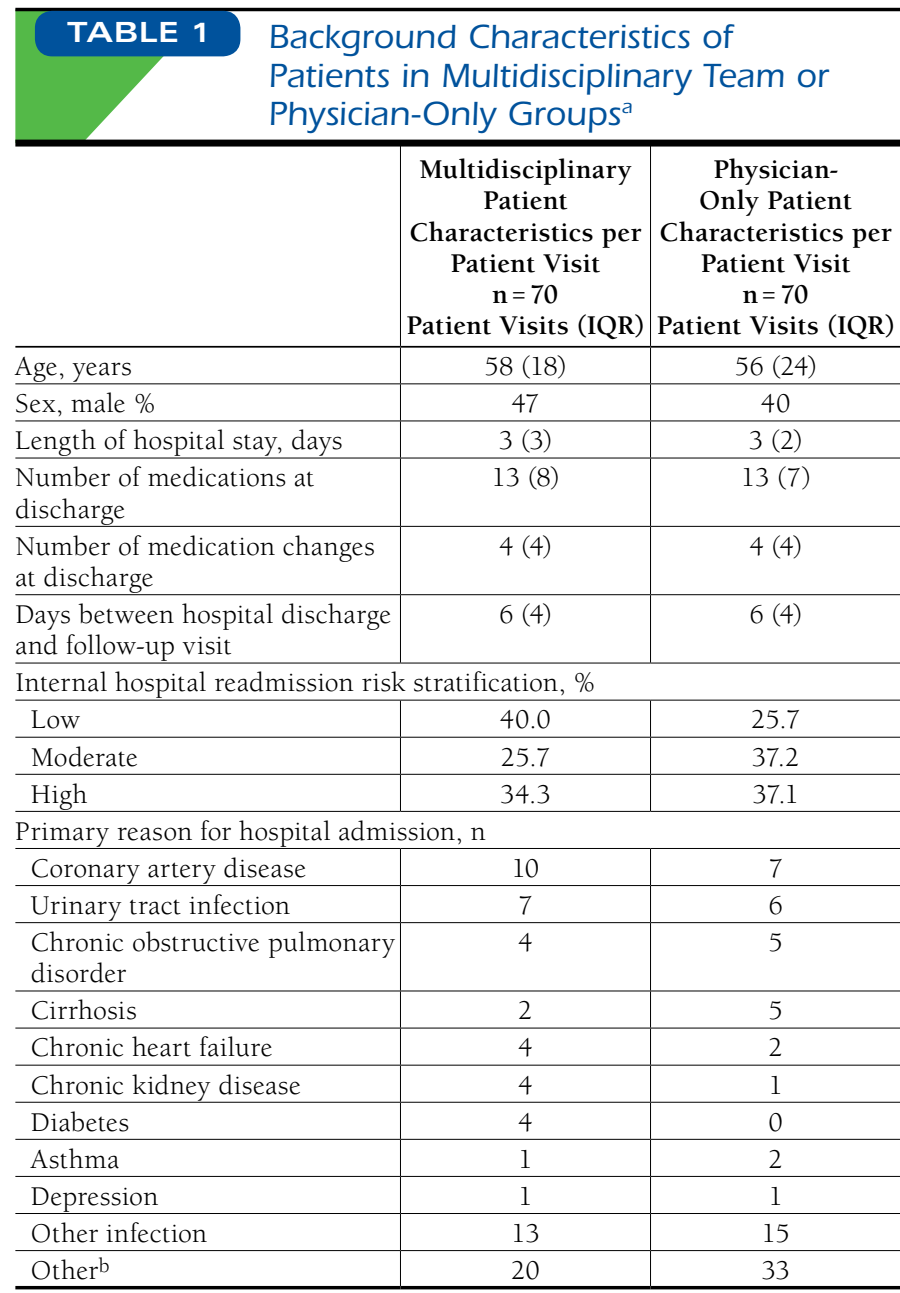

avalues are presented as median.

${ }^{b}$ Other cases include (number in multidisciplinary group, number in physician-only group): trauma $(0,1)$; cancer $(0,2)$; seizure $(0,3)$; atrial fibrillation $(1,0)$; stroke $(2,0)$; electrolyte imbalance $(1,1)$; hemorrhage $(2,0)$; blood clot $(2,1)$; altered mental status $(3,2)$; endocrine disorders other than diabetes $(1,0)$; gastrointestinal disorders $(5,8)$; and chest pain, noncardiac $(3,0)$.

$I Q R=$ interquartile range.

tidisciplinary team during the study period and 52 patient visits completed by the physician-only team were included. Four patients seen by the multidisciplinary team compared with 20 in the physician-only team experienced a 30-day readmission ( $9.5 \%$ multidisciplinary team vs. $38.5 \%$ physician-only team). This difference in readmission rate between groups was even greater than that seen in the primary analysis.

\section{Discussion}

Patients seen by the multidisciplinary team had a $58.3 \%$ relative risk reduction of 30-day hospital readmission compared with those seen by the physician-only team. The multidisciplinary team was successful in reaching the 
program's goal of reducing hospital readmission rates from the original rate of $19 \%$ to $14.3 \%$. These results suggest that the addition of a clinical pharmacist to a hospital follow-up team in a primary care hospital follow-up clinic may improve the transition of care and reduce readmission rates.

\section{Limitations}

Data collection may have been impacted by documentation variability between providers. The same CPP conducted all of the multidisciplinary team visits, while multiple medical residents conducted the physician-only team visits, which may have led to lack of consistency in carrying out the designated components of the visit or variability in documentation. However, the pharmacist individually trained each medical resident on the hospital follow-up clinic visit components, provided a standard visit template that outlined the components, and provided a standard documentation format. In addition, the same attending physicians, who were familiar with the process, worked with the CPP and the residents in an attempt to mitigate variability between the 2 teams.

This study was not designed to match patients based on the internal hospital readmission risk stratification. This led to differences between the 2 groups, with the physician-only team having a higher-risk population. The subgroup analysis, however, suggests that a difference in 30-day readmission rates between the 2 team types exists even when low-risk patients are excluded from both groups. The use of convenience sampling and attempts to match patients based on discharge date may have led to bias in the results. The matches were carried out without knowledge of patient outcomes to reduce the chance of bias. Randomized selection of patients among the 2 visit types could mitigate the chance of bias in future studies. This study only looked at medications and adherence as markers of improvement in the transition of care process. Additional studies are needed to examine any association between 30-day hospital readmission rates and frequency of referrals, adherence with evidence-based care, test follow-up, and medication education.

Based on the positive experience of this program, CPPs within the UNC health care system have been integrated in hospital follow-up clinics in other specialty areas.

\section{Conclusions}

Patients seen after hospital discharge by the multidisciplinary hospital follow-up team had a decreased risk of 30-day hospital readmission. The multidisciplinary team discontinued and initiated medications more frequently as well as addressed nonadherence more often. We hypothesize that these medication interventions were associated with the reduction in 30-day hospital readmission rates. This study supports the involvement of a clinical pharmacist as part of a multidisciplinary team in primary care hospital follow-up visits to improve the transition of care from hospital to home.

\section{Authors}

JAMIE J. CAVANAUGH, PharmD, CPP, BCPS, is Assistant Professor of Medicine, University of North Carolina (UNC) School of Medicine; Assistant Professor of Clinical Education, UNC Eshelman School of Pharmacy; and Clinical Specialist, UNC Hospitals Department of Pharmacy, Chapel Hill. KIMBERLY N. LINDSEY, PharmD, is Pharmacist, Kaiser Permanente, Colorado. BETSY B. SHILLIDAY, PharmD, CDE, CPP, BCACP, is Associate Professor of Medicine, UNC School of Medicine; Associate Professor of Clinical Education, UNC Eshelman School of Pharmacy; and Assistant Medical Director, UNC Internal Medicine Clinic, Chapel Hill. SHANA P. RATNER, $M D$, is Clinical Assistant Professor of Medicine, UNC School of Medicine; Medical Director, UNC Internal Medicine Clinic; and Physician Champion, AccessCare North Carolina, Chapel Hill.

AUTHOR CORRESPONDENCE: Jamie J. Cavanaugh, PharmD, CPP, BCPS, 5034 Old Clinic Bldg., CB 7110, University of North Carolina, Chapel Hill, NC 27599-7110. Tel.: 919.843.0391;

Fax: 919.966.4507; E-mail: Jamie_Cavanaugh@med.unc.edu.

\section{DISCLOSURES}

The authors of this study have nothing to disclose. No financial support was received in order to complete this study.

Study concept and design were contributed equally by Cavanaugh, Shilliday, and Ratner, with assistance from Lindsey. Data collection was primarily conducted by Lindsey, with assistance from Cavanaugh, and all authors participated equally in data analysis. Lindsey and Cavanaugh wrote the manuscript, which was revised by Cavanaugh, Lindsey, Shilliday, and Ratner.

\section{ACKNOWLEDGMENTS}

The authors would like to acknowledge the UNC Chapel Hill Odum Institute for helping with the statistics in this study.

\section{REFERENCES}

1. Tan EC, Stewart K, Elliott RA, George J. Pharmacist services provided in general practice clinics: a systematic review and meta-analysis. Res Social Adm Pharm. 2014;10(4):608-22.

2. Margolis KL, Asche SE, Bergdall AR, et al. Effect of home blood pressure telemonitoring and pharmacist management on blood pressure control: a cluster randomized clinical trial. JAMA. 2013;310(1):46-56.

3. Henry TM, Smith S, Hicho M. Treat to goal: impact of clinical pharmacist referral service primarily in diabetes management. Hosp Pharm. 2013;48(8):656-61.

4. Jencks SF, Williams MV, Coleman EA. Rehospitalizations among patients in the Medicare fee-for-service program. N Engl J Med. 2009;360(14):1418-28.

5. Colivicchi F, Bassi A, Santini M, Caltagirone C. Discontinuation of statin therapy and clinical outcome after ischemic stroke. Stroke. 2007;38(10):2652-57.

6. Hohl CM, Abu-Laban RB, Brubacher JR, et al. Adherence to emergency department discharge prescriptions. CJEM. 2009;11(2):131-38.

7. Ho PM, Tsai TT, Maddox TM, et al. Delays in filling clopidogrel prescription after hospital discharge and adverse outcomes after drug-eluting stent implantation: implication for transitions of care. Circ Cardiovasc Qual Outcomes. 2010;3(3):261-66. 
8. Mesteig M, Helbostad JL, Sletvold O, Røsstad T, Saltvedt I. Unwanted incidents during transition of geriatric patients from hospital to home: a prospective observational study. BMC Health Serv Res. 2010;10:1.

9. Shah M, Norwood CA, Farias S, Ibrahim S, Chong PH, Fogelfeld L. Diabetes transitional care from inpatient to outpatient setting: pharmacist discharge counseling. J Pharm Pract. 2013;26(2):120-24.

10. Schnipper JL, Kirwin JL, Cotugno MC, et al. Role of pharmacist counseling in preventing adverse drug events after hospitalization. Arch Intern Med. 2006;166(5):565-71.
11. Bellone JM, Barner JC, Lopez DA. Postdischarge interventions by pharmacists and impact on hospital readmission rates. J Am Pharm Assoc. 2012;52(3):358-62.

12. Cavanaugh JJ, Jones CD, Embree G, et al. Implementation Science Workshop: primary care-based multidisciplinary readmission prevention program. J Gen Intern Med. 2014;29(5):798-804. 